\section{Opt-on loupes and lights}

Trycare, which has been appointed UK distributor for the complete range of Orange Dental Opt-on loupes and lights, believes that purchasing loupes does not need to be a hassle.

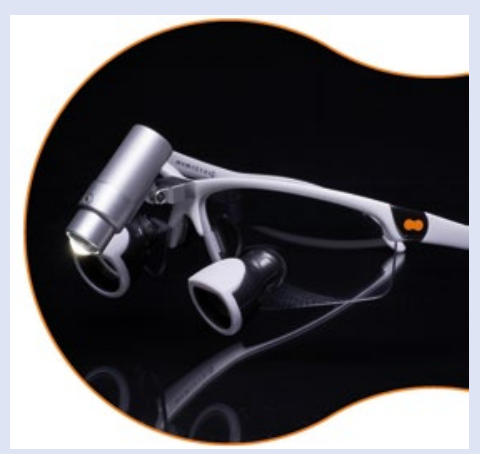

Buying loupes from other companies can be a long-winded procedure involving at least one long surgery visit, lots of time-consuming measuring and a long delay waiting for the loupes.

In addition, if there is a problem, the loupes have to be returned to the manufacturer for correction. Similarly, if components become worn or the operator's prescription changes, they have to be returned for repair or alteration, which can be expensive and take weeks.

Opt-on loupes and lights have a stylish and robust modular design which means they can be easily modified or repaired without returning them to the manufacturer.

If the operator wears glasses and their prescription changes it is easy to do. If the operator wants to switch from glasses to lenses they can, simply by putting in or taking out a prescription adaptor. Incredibly light - just $32 \mathrm{~g}-$ Opt-on loupes are comfortable to wear all day long.

Available in a choice of $2.7 \mathrm{x}$ full arch and 3.3x three-quarter arch variants, in a wide range of colours, they have adjustable arms for enhanced comfort and are supplied in six working distances from $25 \mathrm{~cm}$ to $50 \mathrm{~cm}$.

To complement Opt-on loupes, Trycare offers a choice of wired and cord-free Spot-on lights.

More information is available by visiting https://www.trycare.co.uk/orange-dentalloupes or calling 01274885544 .

\title{
New composite launched for chair-side restorations
}

COLTENE is launching its new BRILLIANT COMPONEER product at the British Dental Conference and Dentistry Show 2019 on 17 and 18 May 2019 in Birmingham.

\section{BRILLIANT COMPONEER is a}

composite material that offers high gloss retention, excellent polishability and outstanding stability. It can be used for minimally-invasive, quality restorations that can be carried out chair-side - no laboratory necessary - in one session.

Its range of indications include restoring carious defects, fractures and old restorations, reconstructing lost tooth substance, extending incisal edges, cosmetic corrections and more.

BRILLIANT COMPONEER is the latest addition to COLTENE's high-performance, cost-effective range of solutions for restorative, endodontic and general dentistry.

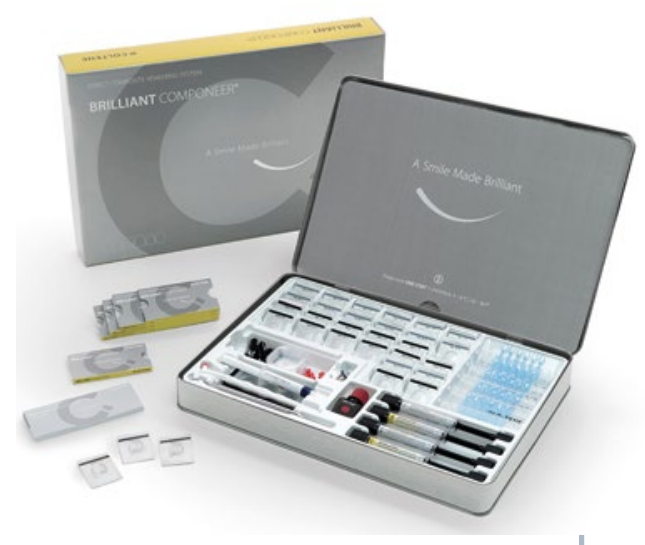

It can be seen at the conference by visiting COLTENE at stand K52.

More information is available by visiting www.coltene.com, emailing info.uk@coltene. com or calling 01444235486.

\section{Words of wisdom with the confident dentist}

Wisdom and The Confident Dentist have announced their new collaboration working together on a highly innovative educational campaign to support dental professionals to encourage habit change in patients.

Experts in oral care and working for improved oral health, Wisdom is dedicated to delivering leading-edge products across the UK, meeting all of a family's oral hygiene needs.

Agreeing with this commitment, Dr Barry Oulton, a practising dentist and founder of The Confident Dentist Academy, is a long-term customer of Wisdom and has been recommending Wisdom Oral Care products to his patients for more than 20 years.

He believes that the key to maintaining a successful at-home oral care regime is through the development and encouragement of habit change in patients by reinforcing and embedding unconscious patterns of behaviour, developed through frequent and positive repetition.

Working together represents the ideal evolution for both parties in this educational endeavour, since The Confident Dentist offers a set of teachable techniques to help dentistry professionals improve the way they communicate with patients, while Wisdom, provides the 'tool kit' to execute habit change.

Combined, they offer a powerful tool in helping to support your patients in achieving

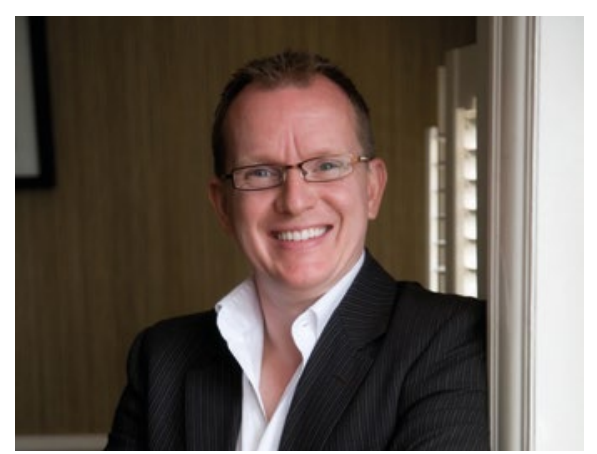

their long-term oral care goals.

A webinar dedicated to habit change, presented by Dr Oulton, will be available later in the year.

In addition, further topics that will be covered during this partnership will include adopting a successful mindset, building rapport with patients, how to use language to best effect, and meta programmes (ie mental processes that manage, guide and direct other mental processes), as well as further information on habit formation and change.

Dr Oulton will be speaking on these issues at the British Dental Conference and Dentistry Show 2019 on 17 May 2019 and the BSDHT Conference on 22 November 2019 in a presentation entitled 'The Wisdom of Great Oral Hygiene Habits'.

For more information, visit https:// wisdom-toothbrushes.com and https://www. theconfidentdentist.com. 